\title{
X-ray microtomography for characterisation of cracks in iron ore pellets after reduction
}

\author{
E. Hjortsberg*1 ${ }^{1}$, F. Forsberg ${ }^{2}$, G. Gustafsson ${ }^{3}$ and E. Rutqvist ${ }^{2,4}$
}

This work presents a method, based on X-ray microtomography and three-dimensional (3D) image analysis, of characterising and quantifying crack distribution in iron ore pellets. The aims have been to verify the method and to determine to what extent crack propagation contributes to the decrease in compressive strength that occurs during reduction at $500^{\circ} \mathrm{C}$ as haematite transforms into magnetite. Raw materials known to cause disintegration problems were selected in order to promote crack propagation. Pellets displayed crack lengths of sizes roughly corresponding to half the pellet diameter already before reduction and, during reduction, a further crack propagation of $\sim 50 \%$ occurred. Through estimations by finite element analysis of the crack size and the pellet geometry, it has been possible to determine that this crack growth most likely is a mechanism that contributes to the decrease in compressive strength. The decrease of $~ 90 \%$ that was experimentally determined to occur after 30 min of reduction is, however, too large to be explained by crack propagation alone. The study shows that the proposed techniques allow 3D imaging of iron ore pellets and characterisation of cracks. The scans are non-destructive and can be carried out repeatedly, which allows a specific sample to be studied at different stages during a process. Through future use of the proposed method, our aim is to reach a deeper understanding of the mechanisms behind low temperature disintegration of iron ore pellets and the performance of LKAB olivine pellets inside the blast furnace.

Keywords: Iron ore pellets, Microtomography, Low temperature reduction, Characterisation of cracks, Finite element modelling, Compressive strength

\section{Introduction}

It has been established previously that haematite pellets swell during reduction and that the swelling is connected to a decrease in mechanical strength. ${ }^{1}$ Together with an applied mechanical strain, this can lead to the disintegration of the material. The degree of disintegration during the transformation to magnetite at $500^{\circ} \mathrm{C}$ is used as a pellet quality parameter [low temperature disintegration (LTD) test, according to ISO13930]. Test conditions are designed to simulate the conditions in the upper part of a blast furnace, and the strain caused by the phase transformation is combined with a mechanical strain as $0.5 \mathrm{~kg}$ pellets are tumbled in a rotating furnace for $1 \mathrm{~h}$. The method was developed during the 1970s in order to prevent degradation problems caused by superfluxing additives. ${ }^{2}$ Today, the method is mainly used as a quality assessment instrument by pellet producers to detect under and overfired pellets.

\footnotetext{
${ }^{1}$ LKAB R\&D, Malmberget SE-983 81, Sweden

${ }^{2}$ LKAB R\&D, Box 952, Luleå SE-971 28, Sweden

${ }^{3}$ Division of Mechanics of Solid Materials, Luleå University of Technology, Luleå SE-971 87, Sweden

${ }^{4}$ Division of Process Metallurgy, Luleå University of Technology, Luleå SE97187 , Sweden

*Corresponding author, email erik.hjortsberg@lkab.com
}

Since 1970, the LKAB olivine pellet has been used successfully primarily in northern Europe. ${ }^{3}$ This has been possible despite the fact that the product displays relatively high levels of disintegration at low temperatures during laboratory testing. Probe samples taken from the LKAB experimental blast furnace ${ }^{4}$ have, however, shown that disintegration rates within the furnace are considerably lower, and outstanding pellet performance has been achieved despite the level of disintegration at laboratory scale. $^{5}$ The reason behind this difference needs further clarification. It has been suggested elsewhere that the high production rate of modern blast furnaces suffices to reduce the severity of the problem. ${ }^{6,7}$

The disintegration during laboratory testing can be assumed to be a result of the combined effect caused by stresses from the phase transformation, the mechanical strain and the initial weaknesses in the pellet structure. In order to describe how these factors affect disintegration, there is a need to characterise how initial weaknesses grow into larger cracks leading to the breakdown of the pellet. This is expected to lead to a deeper understanding of when and to what extent variations in pellet quality at laboratory scale can be expected to affect blast furnace performance.

The aim of the current study is to investigate the possibility of using X-ray microtomography (XMT) and image analysis for quantitative analysis of cracks within 
iron ore pellets during reduction. In addition, an initial approach of using finite element (FE) methods for the modelling of material strength in the pellet, with respect to crack length $(\mathrm{CL})$, is carried out. X-ray microtomography is a non-destructive imaging technique that allows repeated imaging of the full three-dimensional (3D) material microstructure, coded in density. With this technique, it is possible to characterise changes in density as samples are subjected to thermal or mechanical treatments.

This approach has been used for in situ observations of a number of different materials and processes. ${ }^{8,9}$ For example, it has been used for studies of metal powder compacts during sintering ${ }^{10}$ and for analysis of crack propagation in cast iron due to fatigue. ${ }^{11}$ Moreover, a similar methodology has also been used for 3D analysis of structural deformations in various materials, such as bone tissue, ${ }^{12}$ wood, ${ }^{13}$ rock, ${ }^{14}$ cast iron $^{15}$ and granular beds, ${ }^{16}$ by combining XMT imaging with a technique called digital volume correlation. ${ }^{12}$

The fact that XMT gives a $3 \mathrm{D}$ representation of the material makes it a beneficial tool for imaging and analysis of cracks, which, due to their lack of isotropy and symmetry, are hard to predict and model based on single cross-sectional images. Many imaging techniques require the specimen to be sectioned and polished before examination, and the preparation itself might introduce new defects or cause further propagation of existing cracks.

The use of 3D image analysis on the results from XMT makes it possible to identify the cracks inside the material and make a quantitative characterisation of these features. This approach has been used on several other materials and processes, for example, on granular beds, ${ }^{17,18}$ rocks and mineral systems. ${ }^{19-23}$ Moreover, it has been used for studies of pore structure and porosity in iron ore sinter. ${ }^{24,25}$ A review of different applications in the field of soil science is given by Taina et al. ${ }^{26}$

\section{Material and methods}

\section{Material properties and processing Raw material and pelletisation}

Two magnetite concentrates were used in this investigation. The main differences between these are outlined in Table 1 . The first concentrate has been identified previously as a cause for increased deterioration rates during reduction due to its distribution of magnesium within the magnetite lattice. It was chosen to assure that the deterioration of the material would be sufficient for the investigation. The second concentrate was the standard pellet feed from the LKAB Malmberget mine. ${ }^{27}$ Concentrates were mixed with additives (olivine, quartzite, limestone and bentonite) to reach the predetermined specifications and balled on a rotary disc to pellets with sizes of $10-12 \mathrm{~mm}$. Pellets were loaded and indurated in a shaft furnace with a batch size of

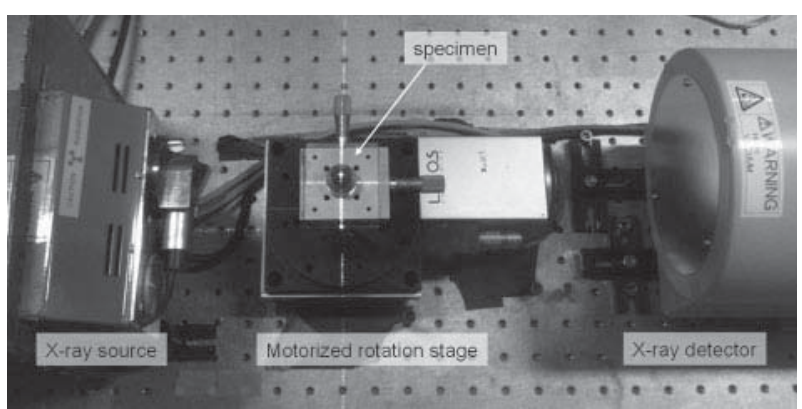

1 X-ray microtomography system

$40 \mathrm{~kg}$. The maximum temperature during induration was $1300^{\circ} \mathrm{C}$.

\section{Reduction}

For the reduction experiments studied with tomography, the pellets were placed in a furnace for determination of free swelling index (according to ISO 4698) and reduced for 10, 20, 30 and $60 \mathrm{~min}$. Reduction was performed at $500^{\circ} \mathrm{C}$ with a gas composition of $20 \% \mathrm{CO}, 20 \% \mathrm{CO}_{2}, 2 \% \mathrm{H}_{2}$ and $58 \% \mathrm{~N}_{2}$ and a gas flow of $5 \mathrm{~L} \mathrm{~min}^{-1}\left(0^{\circ} \mathrm{C}, 1 \mathrm{~atm}\right)$.

Compression strength was measured after reduction (and tumbling) in an LTD furnace (according to ISO13930). Gas flow, gas composition, treatment temperature and exposure times corresponded to those of the free swelling furnace mentioned above.

\section{Sample set-up}

In this investigation, 30 pellets, six unreduced pellets and six at each of the four degrees of reduction were scanned with XMT. The heat treated pellets were only scanned after reduction, which means that no information about the crack distribution in the original, unreduced pellets is available. The advantage of using this approach is that the average crack distribution of six pellets is evaluated instead of relying on a single pellet.

\section{X-ray microtomography}

Figure 1 shows the XMT systems used in this study. The system includes a microfocus X-ray source (L7901-01) and detector unit (C7876-10) from Hamamatsu Photonics. The iron ore pellet samples are positioned on a motorised rotation stage (Linos Photonics, RT120 ST), which, together with the image acquisition, is controlled from a host computer.

During a scan, $744 \mathrm{X}$-ray projection images were collected at equal angles as the sample makes one full rotation. The X-ray tube voltage and current were held at $70 \mathrm{kV}$ and $90 \mu \mathrm{A}$ respectively. The source sample and source detector distances were 8.9 and $31.6 \mathrm{~mm}$ respectively, resulting in a $\times 3.6$ magnification and a theoretical pixel size of $30 \mu \mathrm{m} / \mathrm{pixel}$. The dimensions of the projections were $640 \times 509$ pixels, and the acquisition time was $2 \cdot 56 \mathrm{~s}$.

Table 1 Chemical composition (\%) for magnetite concentrates and pellets used for tomography analysis (grade 1) and compression tests (grade 2)

\begin{tabular}{|c|c|c|c|c|}
\hline & $\mathrm{Fe}$ & $\mathrm{SiO}_{2}$ & $\mathrm{CaO}$ & $\mathrm{MgO}$ \\
\hline Iron ore concentrate, grade 1 & 71 (magnetite) & $0 \cdot 3$ & 0.2 & 0.5 \\
\hline Iron ore concentrate, grade 2 & 71 (magnetite) & $0 \cdot 4$ & $0 \cdot 1$ & 0.3 \\
\hline Pellet composition (both grades) & 67 (haematite) & $2 \cdot 0$ & 0.5 & $1 \cdot 4$ \\
\hline
\end{tabular}




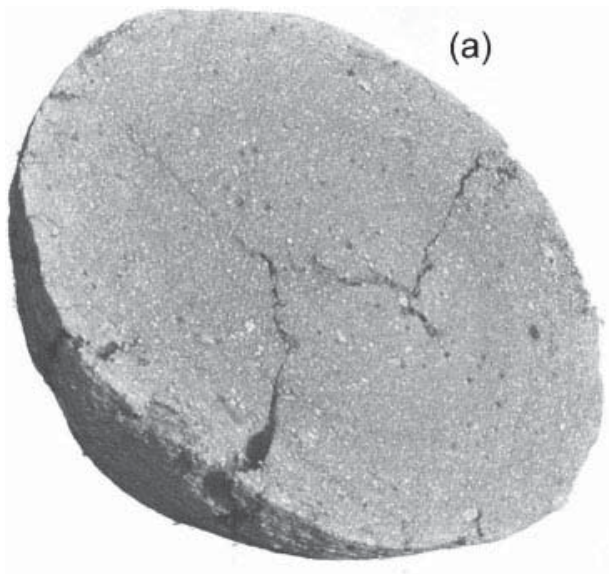

(b)
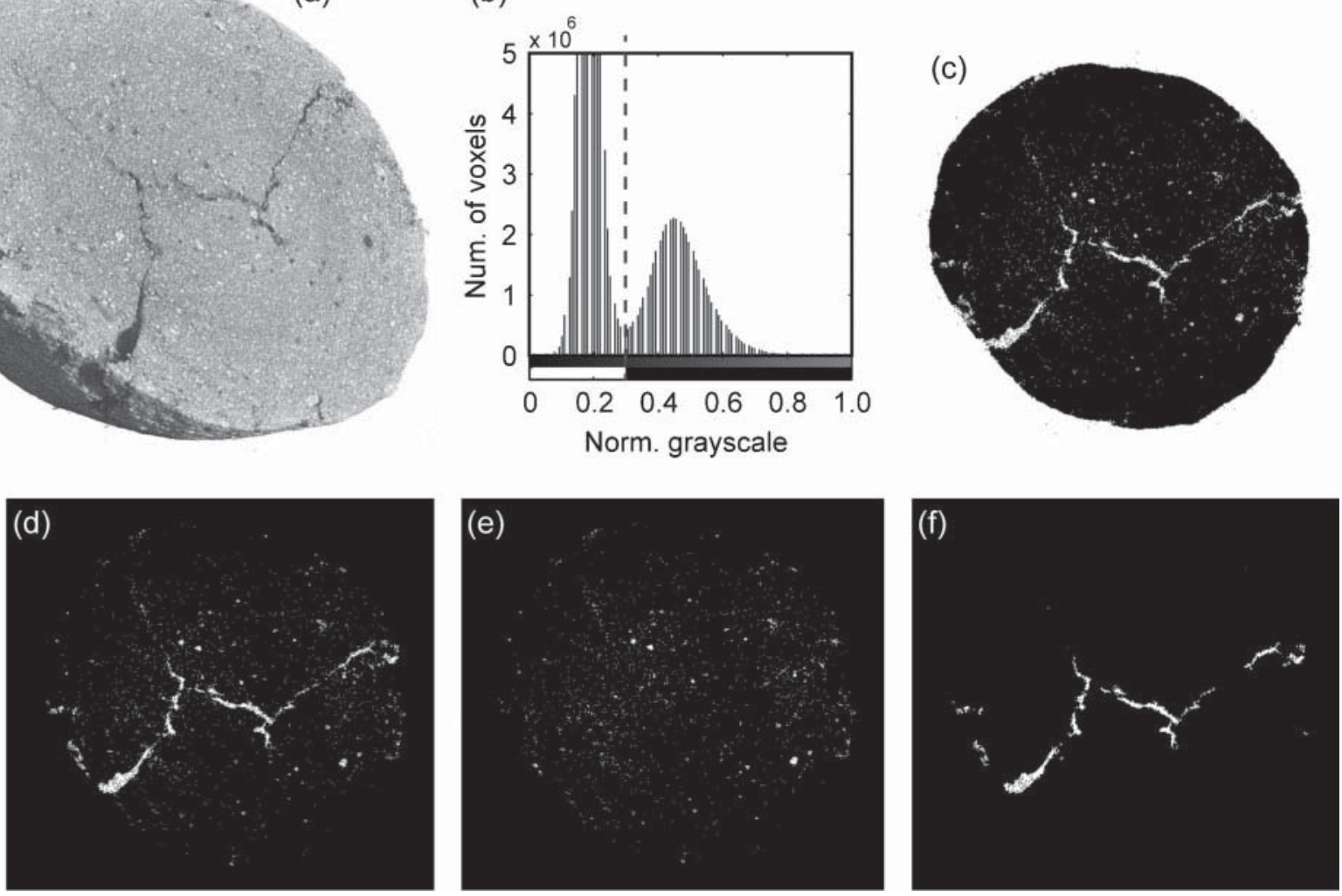

2 a volume rendering of iron ore pellet; $b$ histogram and threshold used for binarisation; $c$ two-dimensional structure after binarisation and $d$ after further subtraction of background; voids visible in $d$ are separated, and $e$ features with close to spherical shape factor (pores and bubbles) are removed; $f$ result from image processing gives features that are categorised as cracks, which is structure upon which further image analysis is based

The tomographic reconstruction was carried out with a cone beam back projection algorithm (Feldkamp type). ${ }^{28}$ Since the results describe the density of the material, the different constituents in the iron ore pellet are represented by different greyscale levels. Regions with high density, for example iron oxide particles, appear bright, while low density regions, like vacant spaces, such as cracks and pores, will appear dark. Before scanning, a white mark was painted on each pellet sample, as shown in Fig. $4 a$ and $d$. The mark represents a simple coordinate system and is aligned with a laser crosshair in the XMT system, as shown in Fig. 1. This enables retrieved information about interior cracks, from scanning and image analysis, to be traced to the outer surface structure.

Hence, this coordinate system enables a rough alignment when performing multiple XMT scans on the same sample or when performing complementary imaging or testing by other techniques.

\section{D image analysis Image processing}

The microstructure of the reconstructed samples was analysed using $3 \mathrm{D}$ image processing and analysis. The software was developed in Matlab using the Image processing toolbox.

The main focus was on the identification and characterisation of the crack network within the material. Figure 2 shows the basic steps of the image processing. Figure $2 a$ shows a volume rendering of a reconstructed iron ore pellet. The scan was performed with a microtomography system from GE. ${ }^{29}$ Figure $2 b$ shows the histogram of the reconstructed 3D data. The dashed line represents the threshold for the binarisation, which was chosen at the minimum value between the histogram peaks $(0 \cdot 15)$. The greyscale values in the left peak correspond to voids in the pellet structure, such as cracks and pores, and also to the reconstructed background (transparent in the volume rendering). The greyscale values at the right hand side of the threshold correspond to the solid state material in the iron ore pellet. In this study, however, the cracks are the features of main interest and therefore the ones filtered out in the binary image (together with other voids and the background). Figure $2 c$ shows the binary structure at the cross-section defined by the cut in Fig. $2 a$. Figure $2 d$ shows the corresponding structure after removal of the background.

A series of morphologic routines were applied in order to highlight elongated structures while suppressing features with a close to spherical shape factor. Figure $2 e$ shows the removed features corresponding to pores and bubbles, and Fig. $2 f$ shows the final structure, where only the crack network is present. Note, however, that these images represent 2D cross-sections, while the actual image processing is carried out on the full $3 \mathrm{D}$ data (the entire reconstructed iron ore pellet).

\section{Feature detection and parameterisation}

In a second step, all remaining features were identified and labelled based on the estimated CL. Here, the length is taken as the Euclidean norm of the smallest box (aligned with the global coordinate system) that entirely encloses the feature, as shown in Fig. 3. Given a 


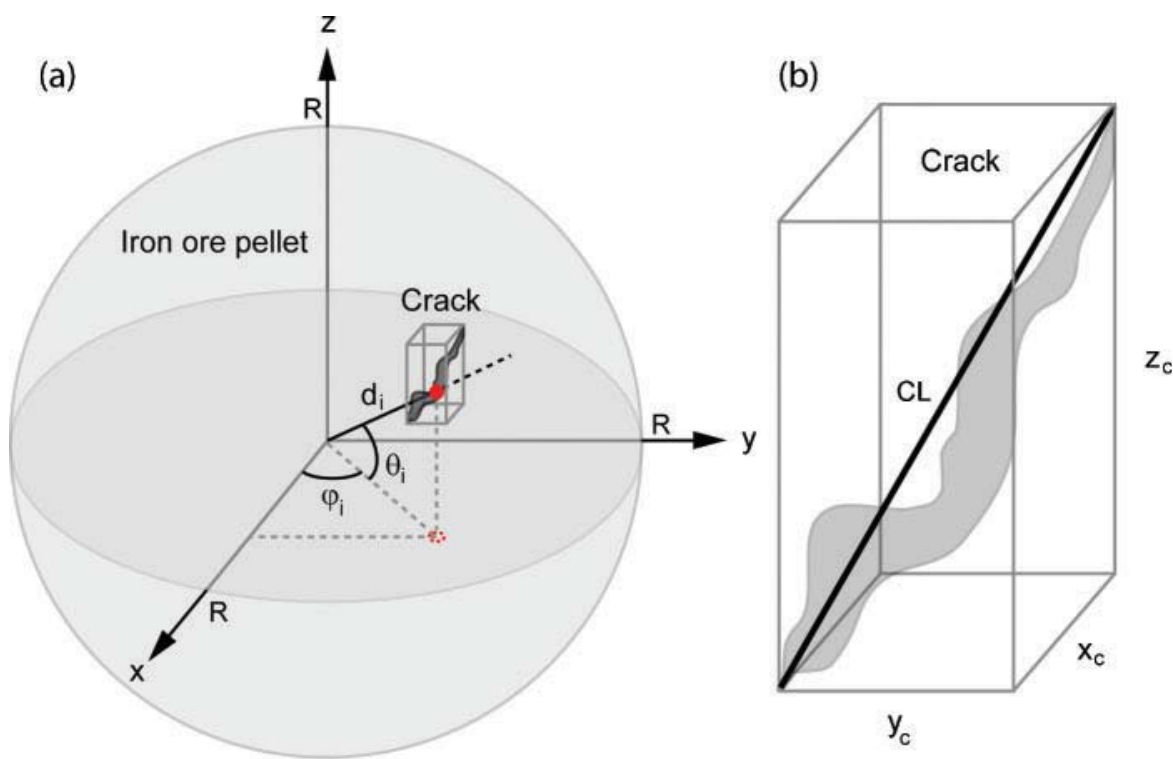

3 a crack analysis is carried out using spherical coordinate system and $b$ crack length is taken as Euclidean norm of smallest box that entirely encloses feature

bounding box with the sides $x_{\mathrm{c}}, y_{\mathrm{c}}$ and $z_{\mathrm{c}}$, as shown in Fig. $3 b$, the estimated CL will be

$$
\mathrm{CL}=\left(x_{\mathrm{c}}^{2}+y_{\mathrm{c}}^{2}+z_{\mathrm{c}}^{2}\right)^{1 / 2}
$$

All parameters associated with spatial quantities such as positions, distances and orientations are determined using a spherical coordinate system, as shown in Fig. $3 a$. The estimated centre position of each crack, which is determined from a centre of mass calculation, is given by the coordinates $d_{\mathrm{i}}, \varphi_{\mathrm{i}}$ and $\theta_{\mathrm{i}}$. The origin in this coordinate system corresponds to the spatial centre of the iron ore pellet, which is also obtained from a centre of mass calculation, under the assumption that the pellet is a homogeneous solid.

Figure $4 a-c$ and $d-f$ shows two different samples represented by photography (Fig. $4 a$ and $d$ ), X-ray microtomography (Fig. $4 b$ and $e$ ) and through 3D visualisation of the crack networks (Fig. $4 c$ and $f$ ). The four largest cracks in each sample are presented in different colours depending on size in descending order: red, yellow, green and blue, using volume rendering

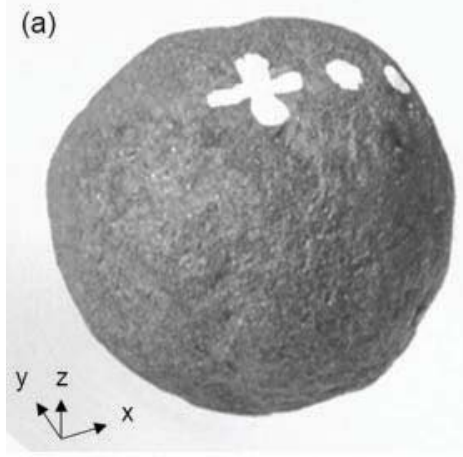

(d)

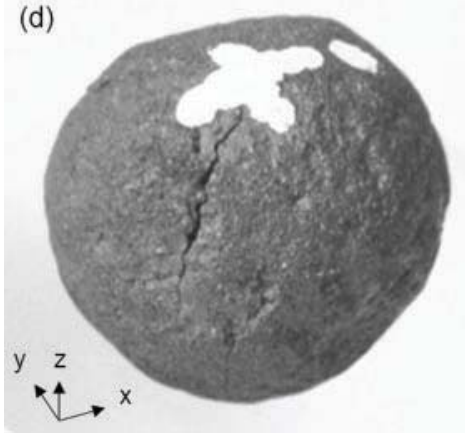

(b)

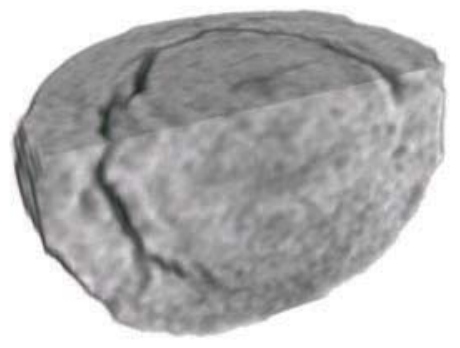

(e)

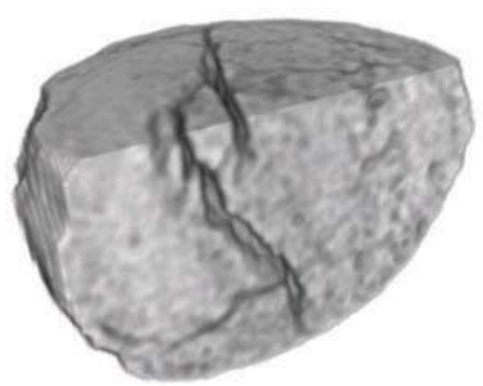

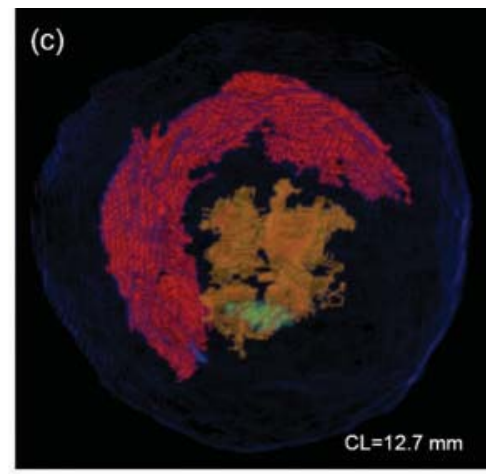

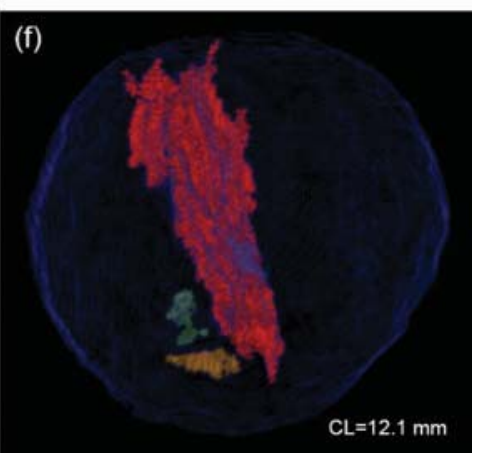

4 Two different types of cracks: $a-c$ images of iron ore pellet that contains major concentric crack and $d-f$ images of another pellet that mainly contain radial crack. $a, d$ photographs; $b$, e tomographic reconstructions; $c, f$ visualisation of 3D crack networks obtained from image analysis. Here, four longest cracks in each sample are shown with different colours, in decreasing order: red, yellow, green and blue. White marks (paint) on samples, shown in $a$ and $d$, represent simple coordinate system and enable retrieved information about interior cracks to be traced to outer surface structure 


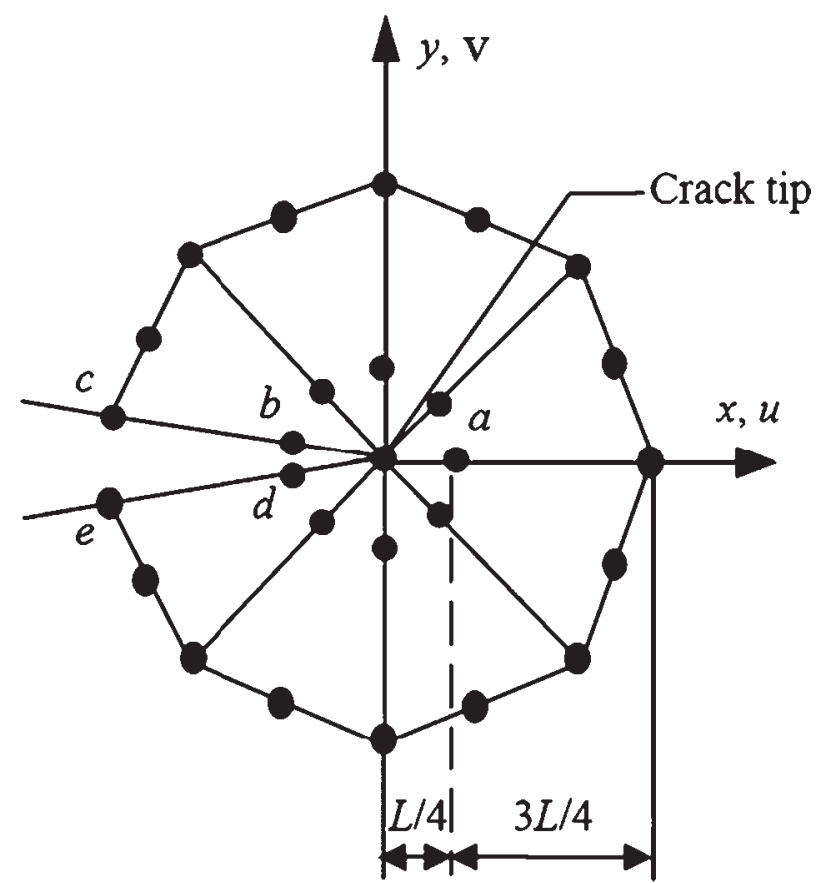

5 Singular quarter point, six-node triangular elements around crack tip

software. $^{30}$ This allows a geometrical interpretation where it is possible to rotate the pellet and observe the crack network geometry from different angles. This will not only give information about the size, shape and position of the cracks but might also reveal possible crack connectivity.

In Fig. $4 c$, the largest crack (red) has a concentric shape, which can be seen in both Fig. $4 b$ and $c$, but not on the surface structure in Fig. $4 a$. The second largest crack (yellow) is radial and found in the centre, oriented orthogonally to the larger concentric crack.

Figure $4 d-f$ shows the same type of plots for a pellet sample that contains a large radial crack, which is clearly visible in Fig. $4 e$ and $f$ and partially reflected on the pellet surface in Fig. $4 d$.

\section{Compression strength measurement}

The pellet compression strength as a function of duration in the LTD test was measured as an average of 20 pellets, which were randomly selected among the non-crushed pellets that remained after each experiment. Pellet compression strength $<100 \mathrm{daN} /$ pellet was measured using a pellet multipress instrument. ${ }^{31,32}$ For levels $>100 \mathrm{daN} /$ pellet, an instrument for determining cold compression strength (according to ISO 4700) was used.

\section{Numerical crack analysis}

Linear elastic fracture mechanics is carried out in order to determine the effect of CL on the compressive two-point loading strength of the pellets. The FE method in the framework of the program package FRANC2 ${ }^{33}$ has been used for simulation of the crack sensitivity. The determination of the stress intensity factor is based on the displacement correlation technique ${ }^{34}$ using singular quarter point, six-node triangular elements around the crack tip (Fig. 5). It is worth noting that in a quarter point element, the mid side node is located at the quarter distance away from the crack tip node in order to provide more accuracy for the calculated stress and displacement fields around the tip. The technique uses the differential displacements of the adjacent nodes across the crack to compute the stress intensity factors. In this work, the stress intensity factor $K_{\mathrm{I}}$ is investigated and computed numerically for the plane strain condition according to

$$
K_{\mathrm{I}}=\frac{E}{8\left(1-v^{2}\right)}\left\{\left(\frac{2 \pi}{L}\right)^{1 / 2}\left[4\left(V_{\mathrm{b}}-V_{\mathrm{d}}\right)-\left(V_{\mathrm{c}}-V_{\mathrm{e}}\right)\right]\right\}
$$

where $E$ is Young's modulus of the material, $v$ is the Poisson's ratio, $L$ is the FE length on the crack face and $V$ are displacements of the FE nodes b, c, d and e.

An idealised 2D FE model of an iron ore pellet with a central straigth crack is worked out. It is a plane strain circular model with a diameter $D$ of $12 \mathrm{~mm}$ and a thickness of $1 \mathrm{~mm}$. Four node elements are used for the outer region of the iron ore pellets; quarter point element for the crack tips and triangular element are used in the region between. A CL sensitivity study is carried out where six different CLs are modelled and solved with an idealised 2D FE model. The FE mesh of the iron ore pellet model is seen in Fig. $6 a$. In Figure $6 b$, a view of the crack is seen in the loaded condition; displacements are magnified 100 times in order to visualise the crack opening. Owing to the lack of material data for iron ore pellets, a phenomenological study is performed where the results are presented in relation to the iron ore pellet with smallest CL. Thus, two arbitrary values of Young's modulus and Poisson's ratio are chosen for the six different CL models.
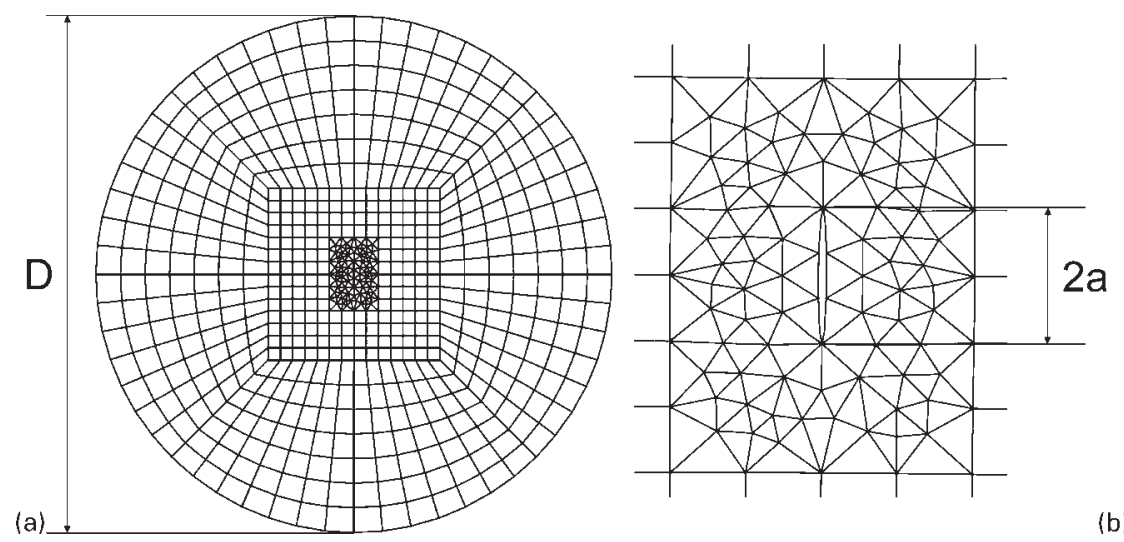

(b)

6 a two-dimensional FE mesh of idealised iron ore pellet with one central crack before loading and $b$ magnified view of the crack opening after loading 

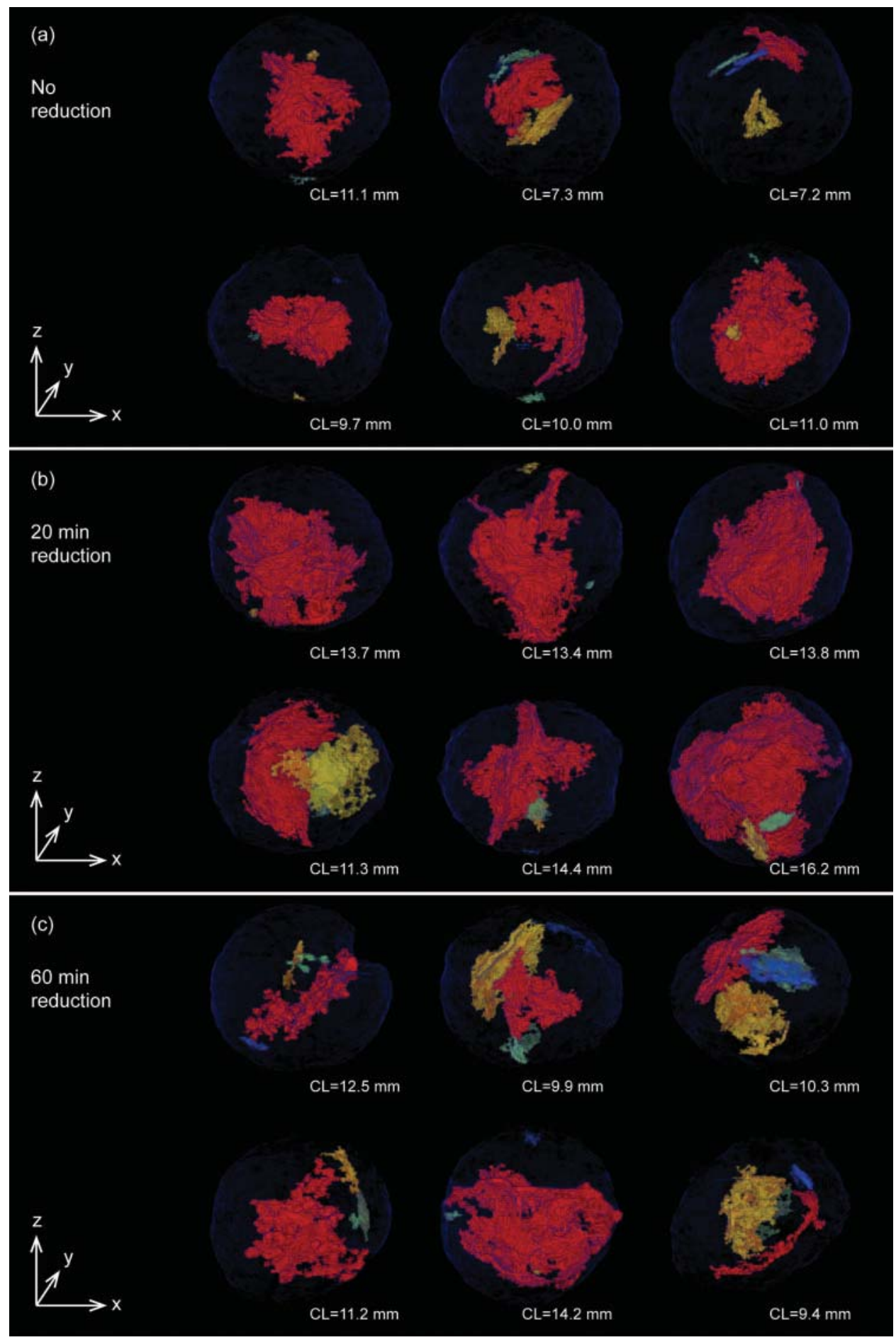

$a$ before reduction; $b$ after $20 \mathrm{~min}$ of reduction; $c$ after $60 \mathrm{~min}$ of reduction

7 Crack network visualisation for 18 different pellets from three reduction states

A distributed load $F$ is applied to the two upper and lower elements of the iron ore pellet model. The load is adjusted to give the same value of the crack intensity factor $K_{\mathrm{I}}$ for all CLs. At an equal value of $K_{\mathrm{I}}$, all cracks are equally strained and likely to break.

\section{Results and discussion}

\section{Crack network visualisation}

Figure 7 shows 3D crack visualisations for non-reduced pellets (Fig. 7a), pellets after reduction for $20 \mathrm{~min}$ (Fig. $7 b$ ) and after $60 \mathrm{~min}$ (Fig. 7c). These images allow a qualitative interpretation of size and geometry for the most prominent cracks.

In the majority of the non-reduced pellets, the largest crack already has a length that exceeds the radius of the pellet. After $20 \mathrm{~min}$, the cracks are significantly larger, and after $60 \mathrm{~min}$, they appear somewhat smaller compared to the cracks in the pellets reduced for $20 \mathrm{~min}$. These results are consistent for all analysed samples. The reason behind the large cracks observed in the unreduced pellets has not been investigated further but might be an indication that the disintegration problems previously experienced with this raw material might originate from effects caused during pellet induration. 


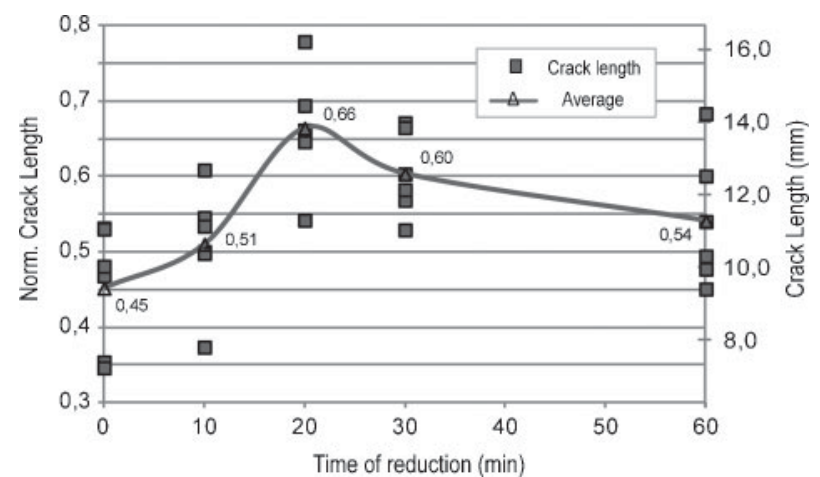

8 Measured $C L$ as function of time during reduction

\section{Quantitative crack analysis}

Figure 8 shows the estimated CL obtained from 3D image analysis as function of the time under reduction for the largest crack in each analysed pellet. On the main $y$ axis, values are normalised to the maximum attainable CL, i.e. the full fracture along the pellet diameter.

The results are consistent with the observations made in the crack network visualisation (Fig. 7), where the largest cracks are observed after 20 min and the samples heat treated for 10, 30 and 60 min display shorter CLs. The CLs after $20 \mathrm{~min}$ are $\sim 50 \%$ longer compared to the cracks in the original unreduced state. The initial increase in CL is expected to be a result of increased stress in the material as the topochemical transformation from haematite to magnetite reaches deeper into the pellet. The observed decrease in CL during the latter part of the heat treatment has not been investigated but could be the result of reduced stress levels as the phase transformation is complete. This could partially close the width of existing cracks below the detection limit of the instrument.

Some of the CL values (displayed on the second $y$ axis in Fig. 8) are longer than the diameter of the pellet itself. This is an effect of the approximation, where CL is obtained from the Euclidean norm (equation (1)) of the smallest box that entirely encloses the crack. As can be seen in Fig. 7, the crack geometry is sometimes rather complex and extends in all three dimensions, which might result in boxes where the space diagonal exceeds the pellet diameter. Normalisation is carried out with respect to the maximum value of CL that is obtainable, which corresponds to a (hypothetical) crack that spans

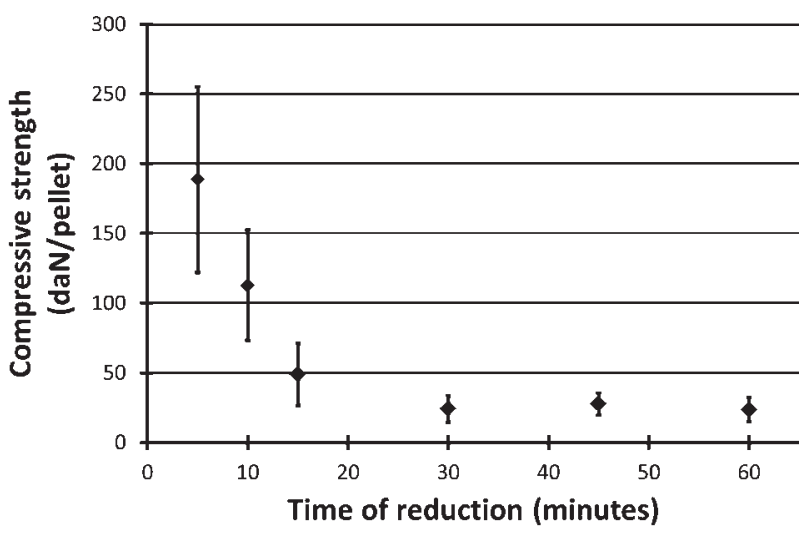

9 Compressive strength for pellets reduced for between 5 and $60 \mathrm{~min}$ : each point shows average and standard deviation for 20 measurements

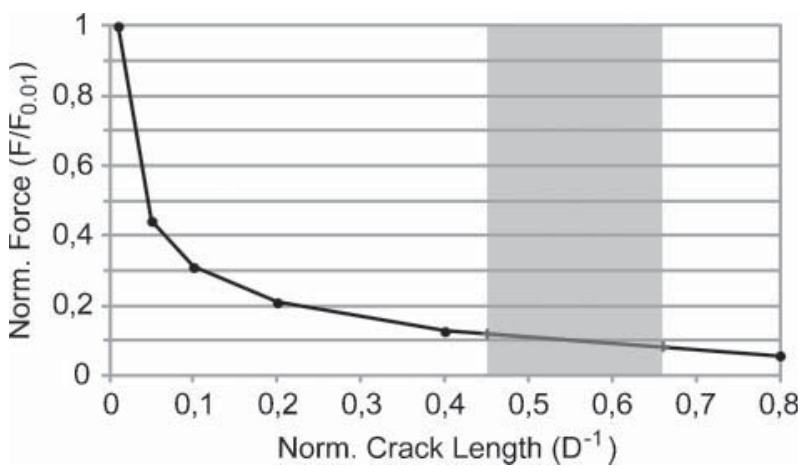

10 Finite element results of normalised force as function of normalised CL at constant crack intensity factor $K_{\mathbf{l}}$

the full diameter of a $12 \mathrm{~mm}$ pellet in all three dimensions $\left(\mathrm{CL}_{\max }=3^{1 / 2} \times 12\right)$.

The analysis clearly shows that the method can characterise the crack network in pellets under reduction and quantify the result to the point where the relative effects on compressive strength can be estimated.

\section{Compression strength measurements}

The compressive strengths for pellets reduced for between 5 and $60 \mathrm{~min}$ are shown in Fig. 9. During reduction, strength decreases from 190 daN/pellet after 5 min to levels $\sim 25 \mathrm{daN} /$ pellet for reduction times between 30 and $60 \mathrm{~min}$, which corresponds to reduction in compression strength of $\sim 90 \%$.

For practical reasons, these measurements had to be performed on pellets manufactured from a different raw material (grade 2 in Table 1) than pellets used in the rest of the investigation. The difference in composition is assumed to have no effect on the decrease in compressive strength.

\section{Estimation of effect of crack growth on compressive strength}

In Fig. 10, the result from the FE modelling is illustrated as the relation between the CL and the applied force needed to break the sample. The result has been normalised to the force from a crack with a length of $1 \%$ of the pellet diameter.

The red coloured region corresponds to the CLs determined in the experimental study, as presented in Fig. 8. However, to make the experimental results comparable with the FE analysis, we assume that the maximum deviation due to the $\mathrm{CL}$ estimator can be treated as a bias and that the normalised CL in Fig. 8 is comparable to that from modelling (Fig. 9). Using this assumption, the experimentally determined CLs (average) span a region from $0 \cdot 45 \mathrm{D}$ to $0 \cdot 66 \mathrm{D}$, a region in which the resulting load and the compression strength scale linearly with the CL.

This indicates that the experimentally observed crack growth of $50 \%$ should correspond to a decrease in compression strength of $\sim 30 \%$. This is less than the approximate $90 \%$ decrease that was determined experimentally (Fig. 9) but within the same order of magnitude. This is interpreted as an indication that crack propagation is one, but most likely not the only, mechanism contributing to the decrease in compressive strength of pellets during low temperature reduction.

The other mechanism likely to be contributing to the decrease in compressive strength is the formation of 
microporosities created as a result of the phase transformation from haematite to magnetite. Previous authors have identified this factor as the main cause for the decrease in compressive strength. ${ }^{35-37}$

\section{Conclusions}

With the equipment and proposed experimental techniques, it has been possible to characterise the crack network in pellets under reduction and quantify the result to the point where the relative effects on compressive strength can be estimated. The analysed pellets contained relatively long cracks of sizes comparable to the pellet radius before reduction. During reduction, a further $50 \%$ increase in CL occurred. With FE modelling, it has been possible to estimate the effect of the crack propagation on compressive pellet strength. The predicted effect roughly corresponds to $30 \%$ of the experimentally determined decrease in strength. This is interpreted as an indication that crack propagation is one, but most likely not the only, mechanism contributing to the decrease in compressive strength of pellets during low temperature reduction.

The main intention has been to develop a method able to characterise crack propagation during reduction, from small defects $(>1 \mathrm{~mm})$ to cracks large enough to cause breakdown of pellets into smaller fragments. The experimental conditions have not been optimal for this purpose, as the analysed pellets were found to contain large cracks before reduction started. Crack propagation during reduction has also been limited and has not in any case been sufficient to cause breakdown into pellet fragments. In future investigations, attempts will be made to apply experimental conditions more similar to those standardised under ISO13930 in order to characterise a larger part of the crack propagation.

\section{Acknowledgements}

Financial support from Hjalmar Lundbohm Research Centre for the work of the third author is gratefully acknowledged. We would also like to thank H.-A. Häggblad and P. Jonsén at the Division of Mechanics of Solid Materials at Luleå University of Technology for their involvement in this work.

\section{References}

1. K. Meyer: 'Pelletizing of iron ores', section $6 \cdot 1 \cdot 2 ; 1980$, Berlin/ Heidelberg/New York, Springer-Verlag.

2. G. Thaning: Ironmaking Steelmaking, 1976, 3, 57-63.

3. M. Hallin: ISS Ironmaking Conf. Proc., 1994, 53, 287-291.

4. A. Dahlstedt, M. Hallin and M. Tottie: Proc. 1st Int. Conf. on 'Process development in iron and steelmaking', SCANMET I, 235-245, MEFOS, Luleå, Sweden, June 1999.

5. A. Brännmark, A. Dahlstedt, C. Stillberg and G. Hyllander: Proc Conf. AISTech 2007, Indianapolis, IN, USA, May 2007, AIST, 315-323.
6. W. Lu, V. Hegde, V. Trofimov and L. Yang: ISS Ironmaking Conf. Proc., 1981, 40, 258-277.

7. B. Hässler: Int. J. Miner. Process., 1974, 1, 193-213.

8. F. Beckmann, R. Grupp, A. Haibel, M. Huppmann, M. Noethe, A. Pyzalla, W. Reimers, A. Schreyer and R. Zettler: Adv. Eng. Mater., 2007, 9, 939-950.

9. J. Buffiere, E. Maire, J. Adrien and E. Boller: Exp. Mech., 2010, 50, 289-305.

10. O. Lame, D. Bellet, M. Di Michiel and D. Bouvard: Nucl. Instrum. Methods Phys. Res. B: Beam Interact. Mater. Atoms, 2003, 200B, 287-294.

11. J. Rannou, N. Limodin, J. Rethore, A. Gravouil, W. Ludwig, M. Baietto-Dubourg, J. Buffiere, A. Combescure, F. Hild and S. Roux: Comput. Meth. Appl. Mech. Eng., 2010, 199, 1307-1325.

12. B. K. Bay, T. S. Smith, D. P. Fyrie and M. Saad: Exp. Mech., 1999, 39, 217-226.

13. F. Forsberg, R. Mooser, M. Arnold, E. Hack and P. Wyss: J. Struct. Biol., 2008, 164, 255-262.

14. N. Lenoir, M. Bornert, J. Desrues, P. Bésuelle and G. Viggiani: Strain, 2007, 43, 193-205.

15. N. Limodin, J. Rethore, J. Buffiere, F. Hild and S. Roux: Acta Mater., 2010, 58, 2957-2967.

16. F. Forsberg and C. R. Siviour: Meas. Sci. Technol., 2009, 20, 095703.

17. G. T. Seidler, G. Martinez, L. H. Seeley, K. H. Kim, E. A. Behne, S. Zaranek, B. D. Chapman, S. M. Heald and D. L. Brewe: Phys. Rev. E, 2000, 62E, 8175-8181.

18. P. Richard, P. Philippe, F. Barbe, S. Bourles, X. Thibault and D. Bideau: Phys. Rev. E, 2003, 68E, 020301.

19. C. Denison, W. D. Carlson and R. A. Ketcham: J. Metamorph. Geol., 1997, 15, 29-44.

20. C. Denison and W. D. Carlson: J. Metamorph. Geol., 1997, 15, 4557.

21. C. L. Lin and J. D. Miller: Int. J. Miner. Process., 1996, 47, 61-73.

22. C. L. Lin and J. D. Miller: Miner. Metall. Process., 2002, 19, 65-71.

23. D. A. Jerram, A. Mock, G. R. Davis, M. Field and R. J. Brown: Lithos, 2009, 112, 223-235.

24. A.-X. Wu, B.-H. Yang, Y. Xi and H.-C. Jiang: J. Cent. South Univ. Technol., 2007, 14, 220-224.

25. V. Shatokha, I. Korobeynikov, E. Maire and J. Adrien: Ironmaking Steelmaking, 2009, 36, 416-420.

26. I. A. Taina, R. J. Heck and T. R. Elliot: Can. J. Soil Sci., 2008, 88, $1-20$.

27. S. Forsmo: Int. J. Miner. Process., 2005, 75, 135-144.

28. A. C. Kak and M. Slaney: 'Principles of computerized tomographic imaging'; 1988, New York, IEEE Press, Inc.

29. GE Measurement \& Control Solutions: Phoenix|x-ray, Phoenix nanotom ${ }^{\circledR}$, http://www.ge-mcs.com/download/x-ray/phoenix-x-ray/ GEIT-31203EN_nanotom.pdf, 2012-08-28.

30. MIPAV, Center for Information Technology (CIT), National Institutes of Health (NIH) and the US Public Health Service. http://mipav.cit.nih.gov/index.php, 2012-08-28.

31. S. P. E. Forsmo: 'Arrangement and method for the analysis of the strength of a specimen of reducible material that contains iron', SE Patent 0500019-5 2005 (US Patent 734104 B2).

32. S. P. E. Forsmo, A. J. Apelqvist, B. M. T. Bjorkman and P. Samskog: Powder Technol., 2006, 169, 147-158.

33. FRANC 2D: version 3·2, http://www.cfg.cornell.edu, 2012-08-28.

34. C. F. Shih, H. G. Delorenzi and M. D. German: Int. J. Fract., 1976, 12, 647-651.

35. K. Meyer: 'Pelletizing of iron ores', section $6 \cdot 1 \cdot 2 \cdot 1$, 1980; Berlin/ Heidelberg/New York, Springer-Verlag.

36. J. O. Edström: JISI, 1953, 175, 289-304.

37. Z. Huang, L. Yi and T. Jiang: Powder Technol., 2012, 221, 284 291. 